\title{
Medicina Baseada em Evidências: Revisão Sistemática com Metanálise como Ferramenta de Tomada de Decisão em Ciências Veterinárias
}

\section{Evidence-Based Medicine: Systematic Review with Meta-Analysis as a Decision-Making Tool in Veterinary Science}

\author{
Francianne Baroni Zandonadia; Fernando Rafael de Mourab; Alexandre Amude*a \\ ${ }^{a}$ Universidade de Cuiabá, Programa de Pós-Graduação Stricto Sensu em Biociência Animal. MT, Brasil. \\ ${ }^{\mathrm{b}}$ Faculdade de Ciências Sociais e Aplicadas de Sinop. MT. Brasil. \\ *E-mail: amamude@hotmail.com
}

\begin{abstract}
Resumo
Em anos recentes, grande quantidade de estudos e de investigações são desenvolvidos, fato que contribui para a divulgação do conhecimento científico e para a tentativa de responder questões que ainda inquietam a sociedade como um todo. Ainda assim, a qualidade das evidências produzidas segue sendo alvo de questionamentos no momento da tomada de decisões, com particular destaque para área da saúde. Nesse cenário, a revisão sistemática com metanálise se configura como ferramenta de grande importância no momento da elaboração de tais diretrizes, em função de qualidade da evidência produzida, por se tratar de um método com regras pré-definidas desde o processo de seleção até a análise dos resultados encontrados. O presente trabalho teve como objetivo elaborar uma revisão narrativa sobre o processo de estruturação das revisões sistemáticas com metanálise. Para tanto, foram realizadas buscas em diferentes bases de dados, utilizando o programa RAYYAN®. Após a adequada seleção dos trabalhos, o programa Mendeley ${ }^{\circledR}$ foi empregado para gerir as referências bibliográficas. Resultados da presente revisão denotam que a revisão sistemática com metanálises constitui a base da estratégia em saúde, denominada de Medicina Baseada em Evidências (MBE), que associa a experiência clínica do profissional às constatações científicas obtidas, prática que contribui muito para a melhoria dos serviços prestados, com destaque para o contexto das Ciências Veterinárias. Diante do exposto, denota-se a necessidade do desenvolvimento de novas revisões sistemáticas, a fim do estabelecimento de uma prática em saúde mais condizente com os achados científicos e focada no aprendizado constante do profissional frente às diretrizes estabelecidas.
\end{abstract}

Palavras-chave: Análise de Resultados. Delineamento. Medicina Veterinária. Metodologia de Pesquisa.

\begin{abstract}
In recent years, a large number of studies and investigations have been carried out, a fact that contributes to the dissemination of scientific knowledge and to the attempt to answer questions that still disturb the society as a whole. Even so, the quality of the evidence produced continues to be questioned when making decisions, with particular emphasis on the health area. In this scenario, the systematic review with meta-analysis is configured as a tool of great importance at the time of drawing up such guidelines, due to the quality of the evidence produced, as it is a method with pre-defined rules from the selection process to the analysis of the results found. The present work aimed to elaborate a narrative review about the process of structuring systematic reviews with meta-analysis. Therefore searches were performed in different databases using the RAYYAN ${ }^{\circledR}$ program. After the adequate selection of papers, Mendeley ${ }^{\circledR}$ was used to manage the bibliographic references. Results of this review show that the systematic review with meta-analysis forms the basis of the health strategy called Evidence-Based Medicine (EBM), which associates the professional's clinical experience with the scientific findings obtained, a practice that contributes greatly to the improvement of the services provided, highlighting the veterinary sciences context. Given the above, there is a need for the development of new systematic reviews in order to establish a health practice which is consistent with scientific findings and focused on professional's constant learning regarding the established guidelines.
\end{abstract}

Keywords: Analysis of Results. Design. Veterinary Medicine. Research Methodology.

\section{Introdução}

A revisão sistemática é uma forma de delineamento de estudos bastante importante para a tomada de decisão, quando se avalia alguma intervenção terapêutica. As revisões sistemáticas possuem o poder de revelar entre vários estudos disponíveis sobre uma determinada temática, aqueles com superioridade metodológica. Para estes estudos, técnicas específicas são utilizadas na identificação de pesquisas originais, com o intuito de minimizar vieses capazes de mascarar os resultados (DONATO; DONATO, 2019).

Resultados diferentes podem, sobremaneira, aparecer em estudos distintos, que abordam as mesmas questões e, estudos isolados, não fornecem evidências estatísticas suficientes para a obtenção de respostas. Sendo assim, a forma de delineamento pela revisão sistemática se torna uma poderosa ferramenta de investigação, influenciando nas decisões, e, com custos menores, quando comparados aos estudos desenvolvidos através de outras metodologias (GALVÃO; RICARTE, 2019).

A diversidade de estudos existentes, atualmente, acerca de muitas temáticas, principalmente, os que envolvem a área da saúde, realizados através de inúmeras metodologias para seu desenvolvimento, dificultam o entendimento e a chegada 
até uma determinada conclusão com relação aos métodos e tratamentos existentes e estudados (DONATO; DONATO, 2019).

A partir disso, o elo entre a pesquisa clínica de qualidade que levanta evidências e que interfere, diretamente, na prática clínica, é denominado de medicina baseada em evidências, a qual se utiliza de provas científicas existentes e disponíveis no momento, com boa validade interna e externa, para a aplicação de seus resultados na condução dos tratamentos e intervenções clínicas (EL DIB, 2007).

Nesse contexto, as revisões sistemáticas se configuram como investigações científicas, definidas através de um protocolo próprio, em que os estudos originais passam a ser considerados como a população do estudo (OKOLI, 2019). Com as revisões sistemáticas, os resultados de estudos primários são sintetizados, diminuindo as chances de erros aleatórios e sistemáticos. Estes estudos, então, são uma excelente ferramenta de apoio à execução da medicina baseada em evidências. Com os resultados sumarizados das revisões sistemáticas, o tratamento estatístico de metanálise poderá ser utilizado, dado o seguimento de regras específicas para essa reunião e análise pela modelagem estatística metanalítica (FERNANDES; SOUZA, 2020).

Extrapolar a utilização desta metodologia de avaliação de resultados de estudos para a área de Medicina Veterinária possui relevância máxima, porque reflete em melhorias para a construção de um saber científico baseado e respaldado em evidências, que direcionam os profissionais aos melhores e mais eficientes e eficazes tratamentos. Dessa forma, este estudo objetivou discutir acerca da elaboração da revisão sistemática com metanálise nas Ciências Veterinárias como uma importante ferramenta de respaldo clínico para a tomada de decisões.

\section{Desenvolvimento}

\subsection{Metodologia}

O presente estudo foi conduzido por meio de revisão literária de trabalhos, nacionais e internacionais, publicados em revistas científicas eletrônicas e impressas, acessíveis por meio da web sobre a revisão sistemática e metanálise. $\mathrm{O}$ programa RAYYAN ${ }^{\circledR}$ foi utilizado para organizar a leitura e seleção dos artigos pelos pesquisadores colaboradores envolvidos. Após a busca sistemática usando o RAYYAN ${ }^{\circledR}$ nas bases de dados, os artigos selecionados para o estudo foram inseridos no programa Mendeley ${ }^{\circledR}$ para a gestão das referências.

Os descritores utilizados foram selecionados da base de dados MeSH do Pubmed, e nas demais bases, sendo essas replicadas em: MEDLINE, EMBASE e Web of Science e as combinações abaixo foram sendo incluídas, com os sinônimos separados pelo termo "OR", aumentando assim a sensibilidade nas primeiras buscas:

1- "systematic review" OR "literature review"
2- "meta-analysis"

3- "veterinary science" OR "animal model"

A busca ocorreu sem fixação de ano e idioma da publicação. Os termos 1, 2 foram pesquisados separadamente e os resultados, de cada uma das buscas, foram combinados usando o termo "AND". O total de 58 artigos foram adicionados ao programa gerenciador de revisões sistemáticas RAYYAN ${ }^{\circledR}$ e, posteriormente, no programa gerenciador de referências bibliográficas Mendeley ${ }^{\circledR}$. As análises nos programas foram realizadas pelos revisores, seguindo os critérios de inclusão e exclusão, e as amostras em duplicata foram excluídas na medida em que os colaboradores (revisores) avançaram nas análises. Os artigos que entregavam elementos para atingir os objetivos do presente estudo foram selecionados para a confecção do presente trabalho.

\subsection{A Revisão Sistemática com metanálise}

As revisões sistemáticas podem ser construídas com um enfoque quantitativo e/ou qualitativo. As revisões sistemáticas quantitativas se utilizam de técnicas estatísticas para a sumarização de resultados provenientes de estudos primários diversos com a ótica em estudos clínicos-epidemiológicos, como: odds ratio (OR), risco relativo (RR), número necessário para tratar (NNT), sensibilidade, entre outros. Dentro da revisão sistemática quantitativa, quando estes resultados, provenientes de diversos estudos, são combinados estatisticamente se tem, portanto, a metanálise (SIDDAWAY; WOOD; HEDGES, 2019). Estes mesmos autores apontam, ainda, que as revisões qualitativas se dividem em narrativas e metassínteses.

As revisões narrativas podem ser empregadas quando os estudos compilados possuem metodologias muito diversas ou quando várias teorias foram conceituadas sobre um mesmo tema, permitindo a síntese de resultados de diferentes estudos quantitativos, sem necessariamente, ter um enfoque no aspecto estatístico dos mesmos. As metassínteses e as metanálises qualitativas são adequadas quando se pretende integrar resultados qualitativos com a finalidade de apontar conceitoschave ou explicações mais convincentes ou inovadoras para determinados eventos, sendo muitas vezes bases para os questionamentos a serem aprofundados nas revisões quantitativas (SIDDAWAY; WOOD; HEDGES, 2019).

A diferença entre a revisão sistemática qualitativa e a quantitativa é, portanto, a sumarização dos resultados. Como nas revisões sistemáticas qualitativas, os dados são apresentados, de forma conjunta, sem a necessidade da sumarização e da preocupação estatística em um primeiro momento, sua evidência é considerada de menor relevância, quando comparadas às pesquisas quantitativas (BRIZOLA; FANTIN, 2016). No entanto, há fatores como heterogeneidade e inconsistência nos resultados, que podem impedir a união dos diferentes estudos sob uma única medida, inviabilizando a realização de uma metanálise adequada(COOK, 1997). 
Sendo assim, a realização dos estudos de revisão sistemática são poderosas ferramentas para a análise e compilação de resultados, com maior robustez, porém a realização da análise metanalítica depende dos tipos de estudos e formas de mensuração e de análises das variáveis executadas nos estudos selecionados (ROEVER, 2017).

\subsection{Critérios para a construção de uma revisão sistemática com metanálises}

Para a construção de uma revisão sistemática com metanálise de qualidade, alguns critérios precisam ser seguidos. Para a evolução do conhecimento, em determinada área de estudo, se faz necessário um grau de cumulatividade de evidências, de forma sistemática e que possa ser usado como evidências que suportem sua indicação. Nessa perspectiva, é primordial estabelecer procedimentos confiáveis e que orientem a síntese de estudos produzidos em uma determinada área de pesquisa (WOLF, 1986). Entre estes critérios para a elaboração deste estudo se tem:

- Delimitar uma questão clínica estruturada. Para a sua elaboração, os autores precisam definir o tipo de paciente, a intervenção, a comparação e o objetivo. Seguir o acrônimo PICO: Paciente (qual situação clínica? Qual é a doença?), Intervenção (qual é o tratamento de interesse a ser testado?), Comparação (grupo controle, placebo, sem ou com intervenção, ou outra intervenção?) e Objetivos (resultados, desfechos clínicos?) (CUMMINGS et al., 2003).

- Realizar uma busca abrangente, pois o ideal é que uma revisão sistemática abarque todas as evidências existentes relacionadas sobre o assunto, entre as diversas bases de dados existentes (Medline, Registro de ensaios clínicos aleatórios da Cochrane Collaboration, EMBASE, LILACS e a verificação das referências citadas em artigos originais e de revisão sobre o tema) (MOHER et al., 1999).

- Descrever, rigorosamente, os critérios de inclusão e segui-los, fidedignamente, (PETROU; KWON; MADAN, 2018).

- Avaliar criteriosamente a qualidade metodológica dos estudos incluídos na revisão sistemática. A inclusão de estudos de baixa qualidade metodológica compromete os resultados da revisão, com a eficácia estatística de alguns estudos deste cunho, ampliada de maneira a mascarar os resultados (HEARST et al., 2003).

- Extração de dados de forma padronizada e sem vieses. Certificar-se de que os revisores estavam cegos com relação à fonte desses estudos, os seus autores e resultados. Padronizar os formulários de coletas de dados e fazer levantamentos prévios dos estudos, pois explorar dados favoráveis à hipótese dos autores pode configurar resultados falso-positivos (ROEVER, 2017).

- Realizar a metanálise. Esta abordagem estatística faz o cruzamento de resultados de estudos relevantes selecionados, respondendo às questões previamente estabelecidas, sintetizando os resultados e sinalizando as áreas e possíveis necessidades de maiores investigações. Os resultados da metanálise só terão de fato relevância se os estudos que a compuserem forem resultantes de uma revisão sistemática, podendo, portanto, produzir estimativas que resumam o todo, denominadas de estimativas metanalíticas (RODRIGUES, 2010).

A necessidade e as tentativas de reunirem os dados resultantes de várias pesquisas, em um só estudo, iniciaram com Karl Pearson, em 1904. Tippet e Fisher, em 1931 e 1932, apresentaram alguns métodos para combinação do valor $p$, e mais tarde, em 1938, Yates e Cochran utilizaram combinação de estimativas obtidas através de experimentos agrícolas. Em 1980, a metanálise passou a ser amplamente utilizada na área de saúde, respondendo questões que envolviam tratamento cardíaco e câncer (WHITEHEAD, 2002).

Nasce em 1993, em Oxford, a Cochrane Collaboration, responsável por organizar, elaborar, manter e divulgar as revisões sistemáticas da área de saúde. Após várias fundações, em diversos locais do Mundo, em 1996, nasce no Brasil o Centro Cochrane, em São Paulo (RODRIGUES, 2010).

Otratamento estatístico utilizado na metanálise é importante para sumarizar a diversidade de resultados existentes em uma medida única, denominada: estimativa de efeito conjunto (COUTINHO, 2003). Para executar a metanálise são utilizados dois métodos: o clínico-epidemiológico de sumarização dos resultados e a quantificação da contribuição dos estudos para análise, muito empregadas na área da saúde.

No parâmetro clínico-epidemiológico é necessário fazer a avaliação dos dados, se esses são binários ou contínuos. Nas variáveis binárias, é necessário observar qual foi a medida de eficácia da intervenção utilizada, se Odds Ratio (OR) ou Risco Relativo (RR), que demostra o impacto clínico. Os dados contínuos devem ser organizados por meio de médias entre os grupos analisados. Nos estudos diagnósticos, os resultados podem ser organizados se observando a sensibilidade, a especificidade e likelihood ratios (PETROU; KWON; MADAN, 2018), enquanto nos estudos de prognósticos são utilizados hazard ratio e medidas de tempo para um evento (BURKE; ENSOR; RILEY, 2016).

\subsection{Modelos de efeitos em Revisões sistemáticas com metanálises}

Em estudos envolvendo a metanálise, dois tipos de modelos são utilizados: o modelo de efeito fixo e o modelo de efeito aleatório. Quando se pressupõe que todos os estudos apontam para um mesmo efeito, e que as diferenças observadas são por erros amostrais, referido na literatura como variabilidade, utiliza-se o método de efeitos fixos (SANTOS; CUNHA, 2013).

Como exemplo podem ser citados os estudos que estão avaliando a eficácia de um medicamento, no qual poderá haver diferenças nos grupos de pessoas selecionadas, com grupos mais saudáveis em um determinado estudo do que em outro, faixa etária variando entre os pacientes dos estudos, classe social, entre outros fatores que podem interferir no efeito do tratamento nos diferentes estudos avaliados (SILVA et al., 2020).

Nesse contexto, quando a heterogeneidade e a diversidade são importantes, sendo preciso utilizar um método denominado de modelo de efeitos-aleatórios, que distribuirá o peso dos estudos de maneira mais uniforme, valorizando a contribuição de todos os estudos, inclusive, dos menores e, neste caso, se 
terá o intervalo de confiança (IC) mais amplo (RODRIGUES, 2010).

Dessa forma, a avaliação da heterogeneidade é fundamental para a escolha do modelo de metanálise, sendo algo a ser feito de forma anterior ao desenvolvimento desse formato de estudo. Os testes Q de Cochran ou pela estatística denominada medida da inconsistência $\left(\mathrm{I}^{2}\right)$ de Higgins e Thompson são as formas mais utilizadas de verificar a presença da heterogeneidade em metanálises (RODRIGUES, 2010).

A heterogeneidade sempre estará presente, sendo assim, antes de apenas atestar sua presença, é preciso fazer a sua quantificação. Então, a medida de inconsistência $\left(\mathrm{I}^{2}\right)$ foi atualmente inserida, que avalia qual o percentual de diferença entre os estudos diferentes, sendo por decorrência de heterogeneidade (HIGGINS et al., 2003). O resultado pode ser exposto em porcentagem, sendo inconsistências de até $25 \%$ consideradas baixas, $50 \%$, intermediárias e, maiores que $75 \%$, altas (BERWANGER, 2007).

Para apresentar os resultados de uma metanálise, usualmente, se utiliza um gráfico denominado Forest Plot, em que os estudos são representados por uma linha, e a combinação dos resultados (metanálise) é representada por um "losango", ou "diamante". Os resultados de cada estudo selecionado são descritos, tanto de forma gráfica (em que os estudos são representados por "quadrados", "círculos" ou outra figura, dependendo do software, cujos tamanhos refletem o "peso" do estudo na metanálise, enquanto os "traços" representam os intervalos de confiança. Quanto maior esta linha, maior é a variabilidade dentro do estudo) quanto de forma numérica. Se os traços do IC ultrapassarem a linha de nulidade, o resultado é estatisticamente não significativo (NEYELOFF; FUCHS; MOREIRA, 2012).

Os estudos maiores, consequentemente, possuem ICs mais estreitos e resultados mais precisos e com maior contribuição para a metanálise. O forest plots apresenta uma vantagem de sumarizar, em um mesmo gráfico, as informações importantes sobre o efeito/precisão do tratamento/intervenção e a contribuição de cada estudo para a análise desenvolvida (BERWANGER, 2007).

Alguns softwares de estatística, como o STATA e o R possuem vários métodos metanalíticos disponíveis. No STATA, as análises são realizadas por meio da linha de comando, que devem ser instaladas para cada análise e condução do estudo. O R é um software estatístico programável, livre e pode ser obtido no site www.r-project.org. A grande vantagem do $\mathrm{R}$ é o fato de ser um software livre. Há disponíveis, também, artigos publicados com guias práticos de execução dos programas de metanálise, sendo um caminho para a execução destas análises, bem como o trabalho em parceria com um estatístico experiente. Para as análises metanalíticas, outras técnicas como a abordagem bayesiana, análises de subgrupo e meta-regressão também podem ser utilizadas (RODRIGUES, 2010).

Para estudos de tratamento e de intervenção, as revisões sistemáticas, com ou sem metanálise, são consideradas nível I de evidência, seguidas de estudos grandes de ensaios clínicos com pacientes (mega trails), com nível II de evidência. Ensaios clínicos, com menos de 1.000 pacientes, possuem nível III, estudos de coorte não randomizados, nível IV de evidências. Os estudos caso-controle e série de casos, níveis V e VI, respectivamente. Os relatos de casos apresentam nível VII de evidência. Já as opiniões de especialistas, pesquisas com animais e as pesquisas in vitro, configuram com nível VIII de evidência, sendo, porém, importantes para a formulação de hipóteses que poderão ser testadas (EL DIB, 2007).

\subsection{Revisões sistemáticas e Ciências Veterinárias}

As revisões sistemáticas, conforme apresentado possuem grande importância na produção de evidências valiosas para a prática clínica, inclusive, em se tratando das Ciências Veterinárias (TOEWS, 2017). Numerosos estudos vêm sendo compilados em revisões sistemáticas à semelhança do que ocorre na medicina humana, proporcionando margem para que os especialistas possam empregar tais conhecimentos em sua atuação profissional (O'CONNOR, 2012).

Contudo, de acordo com Toews (2017) e Grindlay (2015), é digno de nota o fato de que não há uma adequada avaliação da qualidade das revisões sistemáticas produzidas. Muitos dos trabalhos publicados apresentam lacunas graves provocadas por viés, má coleta de dados, fraca ou incompleta revisão da literatura e elaboração incorreta da pergunta de estudo.

De acordo com Sargeant et al. (2010), há uma baixa qualidade, bastante generalizada, dos relatórios clínicos publicados na área veterinária, fato que compromete diretamente a possibilidade de avaliar e replicar tais estudos, em outros grupos de pesquisa, bem como utilizá-los para tomada de decisões clínicas e para a realização de revisões sistemáticas.

Dessa feita, Toews (2017) denota que é necessário um maior rigor por parte dos editores e avaliadores dos periódicos, quando da apreciação e posterior publicação de estudos, a fim da produção de material menos suscetível a desvios que comprometam sua aplicabilidade e confiabilidade. A autora ainda menciona a relevância que o bibliotecário clínico, enquanto especialista na área bibliográfica, possui no momento da adequada seleção de estudos para a busca de uma resposta em pesquisa em saúde e, certamente, em Ciências Veterinárias.

\section{Conclusão}

A quantidade de estudos em medicina veterinária é enorme, com testagens de várias hipóteses diagnósticas em suas mais variadas subáreas. As testagens de métodos, de intervenções e de procedimentos em diversos modelos animais crescem na área veterinária, de forma direta, com o crescimento do mercado pet no Brasil e Mundo. A volumetria de estudos publicados pode dar um norte para as decisões clínicas, porém, se analisadas de forma correta. A modelagem metodológica 
de revisão sistemática é uma poderosa ferramenta para a reunião de diversos estudos, ampliando a amostra submetida aos mesmos tratamentos para um resultado mais consistente e fidedigno.

A possibilidade de realizar as análises metanalíticas com os achados de uma revisão sistemática dão suporte ainda maior aos profissionais que necessitam de um norteador em diversas temáticas para a sua prática clínica, uma vez que a metanálise, enquanto estratégia estatística, ajuda a corroborar os achados da revisão, fortalecendo sua significância e produzindo evidência com alto grau de qualidade. A metanálise é uma ferramenta crucial para o avanço da prática em saúde e, mormente, nas Ciências Veterinárias, em que se pode perceber ainda lacunas em sua empregabilidade.

Sendo assim, produzir estudos que possuam grande nível de relevância e de evidência científica contribuem fortemente para o desenvolvimento da medicina baseada em evidência nas Ciências Veterinárias. Tais estudos embasam as práticas e os procedimentos terapêuticos que envolvem este campo tão amplo, sumarizando e dando suporte aos inúmeros estudos produzidos e publicados. A realização de estudos de revisão sistemática com metanálise em medicina veterinária respaldam as tomadas de decisões e refletem em uma maior segurança às práticas clínicas nesta área, trazendo benefícios aos profissionais e aos usuários dos serviços.

\section{Referências}

BERWANGER, O. Como avaliar criticamente revisões sistemáticas e metanálises? Rev. Bras. Ter. Intensiva, v.19, n.4, p.475-480, 2007. doi: 10.1590/S0103-507X2007000400012

BRIZOLA, J.; FANTIN, N. Revisão da literatura e revisão sistemática da literatura. Relva, v.3, n.2, p.23-39, 2016.

BURKE, D.L.; ENSOR, J.; RILEY, R.D. Meta-analysis using individual participant data: one-stage and two-stage approaches, and why they may differ. Statist. Med., v.36, p.855-875, 2017. doi: 10.1002/sim.7141

COOK, D.J. Systematic reviews: synthesis of best evidence for clinical decisions. Ann. Intern. Med., v.126, p.376-380, 1997. doi: 10.7326/0003-4819-126-5-199703010-00006

COOK, D.J.; SACKETT, D.L.; SPITZER, W.O. Methodologic guidelines for systematic reviews of randomized control trials in health care from the Potsdam Consultation on Meta-Analysis. J. Clin. Epidemiol., v.48, p.167-171, 1995. doi: 10.1016/08954356(94)00172-m

COUTINHO, E.S.F. Meta-análise. In: MEDRONHO, R.A. (Org.). Epidemiologia. São Paulo: Atheneu, 2003. p.447-455.

CUMMINGS, S.R.; BROWNER, W.S.; HULLEY, S.B. Elaborando a questão de pesquisa. In: HULLEY, S.B. et al. Delineando a pesquisa clínica: uma abordagem epidemiológica. Porto Alegre: Artmed, 2003.

DIXON, E.; HAMEED, M.; SUTHERLAND, F. et al. Evaluating meta-analyses in the general surgical literature: a critical appraisal. Ann. Surg., v.241, p.450-459, 2005. doi: 10.1097/01. sla.0000154258.30305.df

DONATO, H.; DONATO, M. Stages for undertaking a systematic review. Acta Med. Port., v.32(3), p.227-235, 2019. doi: 10.20344/ amp. 11923
EL DIB, R.P. Como praticar a medicina baseada em evidências. J. Vasc. Bras., v.6, n.1, p.1-4, 2007. doi: 10.1590/S167754492007000100001

FERNANDES, M.R.; SOUZA, A.D. Medicina baseada em evidência para a tomada de decisão em serviços de saúde: o papel do bibliotecário clínico. Inform. Pauta, v.5, p.36-51, 2020. doi: 10.36517/2525-3468.ip.v5iespecial1.2020.43511.36-51

GALVÃO, M.C.B.; RICARTE, I.L.M. Revisão sistemática da literatura: conceituação, produção e publicação. Logeion, v.6, n.1, p.57-73, 2019. doi: 10.21728/logeion.2019v6n1.p57-73

GRINDLAY, D. Reporting guidelines: how can they be implemented by veterinary journals? Equine Vet. J., v.47, n.2, p.133-134, 2015. doi: 10.1111/evj.12395

HEARST, N. et al. Pesquisa com Dados Existentes: Análise de Dados Secundários, Estudos Suplementares e Revisões Sistemáticas. In: HULLEY, S.B. et al. Delineando a pesquisa clínica: uma abordagem epidemiológica. Porto Alegre: Artmed, 2003.

HIGGINS, J.P.; THOMPSON S.G.; DEEKS, J.J. et al. Measuring inconsistency in meta-analyses. BMJ, v.327, n.7414, p.557-560, 2003. doi: $10.1136 /$ bmj.327.7414.557.

MOHER, D. et al. Assessing the quality of reports of randomised trials: implications for the conduct of meta-analyses. Health Technol. Assess., v.3 I-IV, p.1-98, 1999.

NEYELOFF, J.L.; FUCHS, S.C.; MOREIRA, L.B. Metaanalyses and Forest-plots using a Microsoft excel spreasheet: stepby-step guide focusing on descriptive data analysis. BMC Res. Notes, v.5, n.52, p.1-6, 2012. doi: 10.22239/2317-269x.01514

O'CONNOR, A.M. Improving the quality of reviews in veterinary science: the author's responsibility. Vet. J., v.192, n.2, p.133-134, 2012. doi: 10.1016/j.tvj1.2011.10.014.

OKOLI, C. Guia para realizar uma revisão sistemática da literatura. EaD em Foco, v.9, n.1, e748, 2019. doi:10.18264/eadf. v9i1.748

PETROU, S.; KWON, J.; MADAN, J. A practical guide to conducting a systematic review and meta-analysis of health state utility values. PharmacoEconomics, v.36, n.9, p.1043-1061, 2018. doi: 10.1007/s40273-018-0670-1

RODRIGUES, C.L. ZIEGELMANN, P.K. Metanálise: um guia prático. Rev. Hosp. Clín. Porto Alegre, v.30, n.4, p.436-47, 2010.

ROEVER, L. Understanding systematic review studies. Rev. Soc. Bras. Clin. Med., v.15, n.2, p.127-130, 2017.

SANTOS, E.J.F.; CUNHA, M. Critical interpretation of statistical results of a meta-analysis: methodological strategies. Millenium, v.44, p.85-98, 2013.

SARGEANT, J.M. et al. Quality of reporting of clinical trials of dogs and cats and associations with treatment effects. $J$. Vet. Intern. Med., v.24, n.1, p.44-50, 2010. doi: 10.1111/j.19391676.2009.0386.x

SIDDAWAY, A.P.; WOOD, A.M.; HEDGES, L.V. How to do a dystematic review: a best practice guide for conducting and reporting narrative reviews, meta-analyses, and meta-syntheses. Annu. Rev. Psychol., v.70, p.747-770, 2019. doi: 10.1146/ annurev-psych-010418-102803.

SILVA; J.C.R.A. et al. Clinical trials with drugs in Brazil: an analysis of the main characteristics. Vigil. Sanit. Debate, v.8, n.4, 
p.74-82, 2020. doi: 10.22239/2317-269x.01514

TOEWS, L.C. Compliance of systematic reviews in veterinary journals with Preferred Reporting Items for Systematic Reviews and Meta-Analysis (PRISMA) literature search reporting guidelines. J. Med. Libr. Assoc., v.105, n.3, p.233-239, 2017. doi: $10.5195 /$ jmla.2017.246

WOLF, F.M. Meta-analysis: Quantitative methods for research synthesis. Sage, 1986.

WHITEHEAD, A. Meta-analysis of controlled clinical trials. Jhon Wiley \& Sons, 2002. 